\title{
THE THIRTY-THIRD SUMMER MEETING OF THE AMERICAN MATHEMATICAL SOCIETY
}

The thirty-third summer meeting and eleventh colloquium of the Society was held at the University of Wisconsin, Madison, Wisconsin, from Tuesday to Saturday, September $6-10,1927$, preceded by the summer meeting of the Mathematical Association of America. It will be remembered as one of the most notable occasions in the annals of the Society.

The colloquium lectures by Professors Bell and Wheeler were delivered on Tuesday, Wednesday, Thursday, and Saturday mornings, and Thursday evening. On Thursday afternoon and Friday morning the Society met in sections for the reading of papers, the Sections of Analysis and of Geometry meeting on Thursday and those of Algebra and Analysis and Miscellaneous Subjects on Friday. The joint dinner of the Society and Association with Professor C. S. Schlichter as toastmaster held at the Maple Bluff Golf Club on Wednesday evening was attended by 150 persons.

A reception was tendered the visiting mathematicians and their friends by the officials of the University, and a program of interesting excursions was carried out, and ample provision was made for sports. A picture of the attending mathematicians and their friends was taken, a copy of which is being distributed with this issue of the Bulletin.

The attending mathematicians and their friends were entertained at Chadbourne and Barnard Halls of the University; the meetings were held in Bascom Hall and the colloquium lectures in Sterling Hall.

Resolutions were passed expressing profound appreciation of the cordial hospitality of the University; thanks to the department of mathematics and in particular to the local members of the Committee on Arrangements, Professors E. B. Van Vleck (Chairman), Arnold Dresden, and Warren Weaver; to Professor R. W. Babcock, who was in 
charge of the registration at Chadbourne Hall; and to others who contributed to the success of the gathering.

Many attending members recalled with pleasure the earlier summer meeting and colloquium held at the University of Wisconsin in 1913. On that occasion Professor L. E. Dickson gave a series of lectures entitled "On invariants and the theory of numbers," and Professor W. F. Osgood a series entitled "Topics in the theory of functions of several complex variables." The 1927 colloquium must therefore be known as the Second Madison Colloquium. A special report is given on page 663 of the present issue of the Bulletin.

The attendance included the following one hundred thirty-three members of the Society:

F. E. Allen, Altshiller-Court, W. E. Anderson, Archibald, R. W. Babcock, Barnett, Batchelder, W. O. Beal, E. R. Beckwith, E. T. Bell, Birkhoff, Blichfeldt, Bray, Brenke, Brink, E. W. Brown, H. E. Buchanan, L. H. Bunyan, Bussey, Cairns, C. C. Camp, Carlson, Carmichael, Chang, Chittenden, J. T. Colpitts, Comstock, Conkwright, C. F. Craig, H. V. Craig, Crathorne, Curry, Dalaker, H. T. Davis, Dean, Dickson, Dines, Dowling, Dresden, Emch, G. C. Evans, H. P. Evans, Feinler, W. B. Ford, Fry, Gaba, Garrett, Gergen, Gill, D. C. Gillespie, M. C. Graustein, W. C. Graustein, L. M. Graves, Griffiths, V. G. Grove, Guggenbühl, Harkin, W. L. Hart, W. W. Hart, Hartung, E. R. Hedrick, Hildebrandt, Hoersch, Hollcroft, Hosford, Hotelling, J. C. Hughes, Louis Ingold, Ingraham, Dunham Jackson, Jensen, Kearney, Kempner, Krathwohl, E. P. Lane, Langer, Latimer, Harry Levy, Logsdon, J. V. McKelvey, MacMillan, March, Maria, A.S. Merrill, Michal, Mickelson, Mills, Miser, E. J. Moulton, Olson, F. W. Owens, H. B. Owens, Palmer, Parkinson, Pettit, E. C. Phillips, Phipps, Pierce, Pogo, R. G. D. Richardson, H. L. Rietz, E. D. Roe, Roever, Roos, Roth, Runge, Sanger, Shewhart, Shohat, Sinclair, Skinner, Slaught, Slichter, Snedecor, Virgil Snyder, Stouffer, Swartzel, Tate, J. H. Taylor, E. M. Thomas, Torrey, Trjitzinsky, Van Vleck, Wall, J. H. Weaver, Warren Weaver, Westfall, A. P. Wheeler, Widder, Wolff, Worthington, Yanney, Yeaton.

The Secretary announced the following elections to membership in the Society:

To sustaining membership:

The Georgia Power Company, Atlanta, Ga.;

The University of Michigan, Ann Arbor, Mich.

To ordinary membership:

Dr. Clement Walker Andrews, Librarian, John Crerar Library;

Mr. Loring Beal Andrews, Harvard University; 
Mr. John William Arnold, Western Union Telegraph Co., New York City;

Professor Herman T. R. Aude, Colgate University;

Mr. Benjamin Brownstein, tube mill engineer, Ellwood City, Pa.;

Mr. Arnold Chaimovitch, University of Chicago;

President Harry F. Chapell, Chapell Auditors, New York City;

Professor Edward Everett Colyer, Kansas State Teachers College, Hays;

Professor Walter H. Durfee, Hobart College;

Mr. Theodore Miller Edison, East Orange, N.J.;

Mr. Henry Ericson, Washington High School, Milwaukee;

Mr. John Graham Foley, engineer, New York Central Railroad;

Mr. Edwart T. Frankel, National Industrial Conference Board, New York City;

Mr. Albert R. Gallatin, of Smith and Gallatin, New York City;

Professor Joaquin Gallo, Observatorio Astronomico Nacional, Tacubaya, Mexico;

Professor Horace Newton Hubbs, Hobart College;

Mr. Eugene Law Ickes, geologist, Los Angeles;

Professor Richard P. Johnson, Carnegie Institute of Technology:

Miss Elizabeth E. Knight, Milwaukee State Normal School;

Mr. Joseph Harrison Kusner, University of Florida;

Mr. John Buchanan MacLeod, Chicago;

Dr. James D. Maddrill, Union Labor Life Insurance Company, Washington;

Mr. Phillip Newman, Columbia University;

Mr. William Ross Paul, Chicago;

Professor Thomas Reeve Rosebrugh, University of Toronto;

Mr. Samuel Barnell Rosenbaum, Milford School, Milford, Conn.;

Professor Anestis George Sarafoglou, Robert College, Constantinople;

Dr. Gyan Chand Sharma, University of Notre Dame;

Mr. Hartley L. Smith, New England Power Company, Worcester, Mass.;

Professor Louis Beaufort Stewart, University of Toronto;

Dr. Dirk Jan Struik, Massachusetts Institute of Technology;

Mr. Paul McCartney Swingle, Ohio State University;

Miss Helen Thompson, Psycho-Clinic, Yale University;

Mr. James Edgar Thompson, Pratt Institute;

Mr. Charles Wexler, Roxbury, Mass.;

Mr. George Washington Wishard, Norwood, Ohio;

Mr. Byron C. Wolverton, Friendship Telephone Company, Syracuse;

Mr. Samuel Harry Woolf, Harvard University.

Nominees of sustaining members:

Mr. Charles M. Biscay, Western and Southern Life Insurance Company;

Mr. Charles M. Biscay, Jr., Western and Southern Life Insurance Company;

Mr. Wyman A. Bristol, University of Pennsylvania;

Mr. D. K. Chenoweth, Western and Southern Life Insurance Company;

Mr. C. E. Nelson, Missouri State Life Insurance Company;

Mr. R. A. Ryan, Western and Southern Life Insurance Company;

Professor S. E. Stillwell, Western and Southern Life Insurance Company. 
The following members of the London Mathematical Society have accepted membership under the reciprocity agreement:

Professor William E. H. Berwick, University College, Bangor, Wales; Mr. Charles Henry Rowe, fellow of Trinity College, Dublin.

Twenty-three applications for membership were received.

On recommendation of the Council, the Society adopted two amendments of the by-laws. The first adds to the membership of the Council the three representatives of the Society on the Editorial Board of the American Journal of Mathematics, thus placing this periodical on the same basis as the Bulletin and the Transactions. The second replaces the Assistant Secretary by two Associate Secretaries, with the expectation that the extra official will be in the east.

It was announced that Professor E. W. Brown has accepted the invitation to give the fifth Josiah Willard Gibbs Lecture in connection with the 1927 Annual Meeting in Nashville.

The following appointments were announced: Committee on the Society Visiting Lectureship, Professors G. D. Birkhoff, G. A. Bliss and E. R. Hedrick; Committee on Arrangements for the Gibbs Lecture, Professors W. L. Miser (Chairman) and James McClure of Vanderbilt University and Mr. J. L. Wilson of Nashville; additional representatives of the Society on the augmented American Section of the International Mathematical Union, Professors G. A. Bliss, A. B. Coble, Arnold Dresden, E. V. Huntington, and C. H. Sisam.

On account of the change in his residence Professor Arnold Dresden presented his resignation as Assistant Secretary and a resolution of thanks was passed by the Society as follows:

"Resolved that we, the members of the American Mathematical Society, especially those who have been attending the Chicago or Western meetings of this Society, express to Professor Arnold Dresden our sincere appreciation of and gratitude for the many ways in which he has been of service to these meetings during the last eleven years. We feel that 
his genuine interest in the welfare of the Society, his untiring efforts in its behalf, and the consummate skill with which he has conducted its affairs, including the arrangements for its meetings, in this section of the country have been of inestimable value to the growth of mathematical interest."

Professor M. H. Ingraham was appointed to fill the vacancy for the remainder of 1927 .

Titles and abstracts of the papers read before sectional sessions of the Society follow below: the papers numbers 1 to 8 were read before the Section of Analysis, Professor G. C. Evans presiding; numbers 9 to 16 and 69 before the Section of Geometry, President Snyder presiding; numbers 17 to 30 and 66 to 68 before the Section of Algebra, VicePresident Bell presiding; and numbers 31 to 65 before the Section of Analysis and Miscellaneous Subjects, expresident Birkhoff presiding. Mr. Heineman was introduced by Professor Dresden, Professor Besicovitch by Professor Tamarkin (his paper was read by Professor Langer), Professor Uspensky by the Secretary, and Mr. Dorroh by Professor R. L. Moore. The papers of the following authors were read by title: Ayres, Birkhoff (first paper), Buchanan (second paper), Evans (second paper), Kempner (first paper), Langford, Mears, Merriman, Miller, Moore, Rainich, Schelkunoff, Sharpe, Roos (second paper), Uspensky (second paper), Vandiver, Walsh, Webber, G. T. Whyburn, W. M. Whyburn, Widder and Gergen, Wilson.

1. Professor J. A. Shohat: On certain developments in series of Tchebycheff polynomials.

Consider the finite development $x^{n}=H_{0} \phi_{0}(p ; x)+\cdots+H_{n} \phi_{n}(p ; x)$, where $\phi_{n}(p ; x), n=0,1,2, \cdots$, is a system of Tchebycheff polynomials corresponding to a given interval $(a, b)$ with the characteristic function $p(x)$. In the case $(a, b)=(-1,1), p(x) \equiv 1$ (Legendre polynomials), all the coefficients $H_{i}$ above, as is well known, are positive, a property leading to many interesting results. In the present paper the author extends this property of the $H$ 's to the general case of "symmetric Tchebycheff polynomials": $a=-b$ (finite or infinite), $p(x) \equiv p(-x)$. Application is made, in particular, to the discussion of the convergence of the development $f(x) \backsim \sum_{n=0}^{\infty} A_{n} \phi_{n}(p ; x)$, where the $A_{n} \equiv \int_{-a}^{a} p(x) \phi_{n}(p ; x) f(x) d x$ are all positive and $f(x)$ and $p(x)$ are subject to certain general conditions. 
2. Professor L. L. Dines: Linear inequalities in general analysis.

This paper appears in full in the present number of this Bulletin.

3. Professor L. L. Dines: A theorem on orthogonal sequences.

For any positive constant $e$ greater than unity, we denote by $\mathfrak{S}_{c}^{\prime}$ and $\mathfrak{S}_{e}^{\prime \prime}$ the classes of all infinite real sequences $\left\{\sigma_{i}^{\prime}\right\}$ and $\left\{\sigma_{i}^{\prime \prime}\right\}$ respectively, such that the series $\sum_{i}\left|\sigma_{i}{ }^{\prime}\right| e$ and $\sum_{i}\left|\sigma_{i}{ }^{\prime \prime}\right| e /\left(e^{-1)}\right.$ converge. If $\left\{\sigma_{i}{ }^{\prime}\right\}$ and $\left\{\sigma_{i}{ }^{\prime \prime}\right\}$ are sequences of these respective classes, then the series $\sum_{i} \sigma_{i}{ }^{\prime} \sigma_{i}{ }^{\prime \prime}$ converges absolutely, as is well known. If the sum of this product series is zero, the two sequences are said to be orthogonal. We prove the following theorem: A necessary and sufficient condition that there exist in $\widetilde{S}_{e}^{\prime \prime}$ a positive sequence orthogonal to each of a given finite set of sequences in $\mathfrak{S}_{e}^{\prime}$ is that no linear combination of the given set be $M$-definite.

4. Professor R. E. Langer: The boundary problem asso-. ciated with a differential equation in which the coefficient of the parameter changes sign.

This paper deals with the boundary problem associated with the differential system $u^{\prime \prime}(x)+\lambda x^{v} u(x)=0, u(-\alpha)=u(\beta)=0, \alpha, \beta>0$, in which $\nu$ is any real positive constant. The interest in the system centers on the fact that the interval considered contains the origin, so that the coefficient of the parameter changes sign if $\nu$ is an odd integer, and in general changes from real to complex. The method, that of asymptotic forms, has not heretofore been applied to the study of systems of this type. Asymptotic expressions for the characteristic values and functions are derived, and the theorem for the expansion of an arbitrary function as established is far more general than the existing theorems derived by other methods.

5. Professor W. B. Ford: On the behavior of integral functions in distant portions of the plane.

This paper will appear in full in an early number of this Bulletin.

6. Professor Dunham Jackson: On the approximate representation of analytic functions.

This paper will appear in full in an early number of this Bulletin.

7. Professor T. H. Hildebrandt: Lebesgue integration in general analysis.

This paper points out a method for defining a Lebesgue type of integration in the case of functionals on a linear real range to a complete linear function space. Riemann integration for such functionals has been defined by L. M. Graves (Transactions of this Society, vol. 29 (1927), pp. $166 \mathrm{ff}$.). The method of defining a Lebesgue integration is suggested by the validity 
of Osgood's theorem in this situation, i.e., if a sequence of Riemann integrable functionals bounded in their totality converges to a Riemann integrable functional, then the limit of the integrals is the integral of the limit. Starting with a class of Riemann integrable functionals, e.g., the continuous functionals, and using convergent (or almost convergent) sequences of functionals, we can define integration for functionals not Riemann integrable.

8. Professor T. H. Hildebrandt: Note on interchange of order of limits.

This paper will appear in full in an early number of this Bulletin.

9. Mr. G. A. Parkinson: A theory of parallelism in subspaces.

In this paper the angle between two vectors at different points of a $V_{n}$ is defined. On the basis of this definition, the notion of the parallel motion of a vector in $V_{m_{1}}$, a sub-space of $V_{n}$, with respect to $V_{m}$, also a subspace of $V_{n}$, is developed. The special case in which $V_{m_{1}}$ and $V_{m}$ are applicable is discussed. Parallel curves of $V_{n}$ are defined, and a notion of orders of parallelism between sub-spaces $V_{m_{1}}$ and $V_{m}$ is discussed.

10. Mr.S. A. Schelkunoff: On certain properties of orthogonal and generalized orthogonal transformations.

This paper gives an extension of Euler's formulas for transformation of coordinates, and certain properties of the coefficients, also a decomposition of a general rotation in an $n$-flat into a succession of "simple" rotations, and the determination of the angles of simple rotations. The generalized orthogonal transformations are defined by means of relations between the coefficients $a_{k}{ }^{i}: a_{k}{ }^{i} \bar{a}_{k}{ }^{j}=1$ when $i=j$, and $a_{k}{ }^{i} \bar{a}_{k}{ }^{j}=0$ when $i \neq j$, where $\bar{a}_{k}{ }^{i}$ is the conjugate of $a_{k}{ }^{i}$. Certain properties of generalized orthogonants are then obtained. The remainder of the paper is concerned with quasimetrical transformations of null-flats.

11. Professor T. R. Hollcroft: Limits for double points of surfaces.

Limits that can be reached but not exceeded are found for the number of distinct conic nodes of an algebraic surface of any order. For orders greater than seven, these limits are smaller than those previously found. Also the maximum number of binodes of each kind and unodes of each kind is found for any algebraic surface. It is further shown that all double points of whatever kind that an algebraic surface can have may be real. The maximum numbers of consecutive conic nodes, binodes and unodes of surfaces of given order are determined. Distinct conic nodes account for one invariant each, but when they combine to form a higher singularity, the number of conic nodes is greater than the number of invariants. A certain number of conic nodes may thus be considered independent and the rest dependent. For a given order of a multiple point, a limiting order 
of the surface containing it is found such that for a smaller order of the surface the number of independent conic nodes in the multiple point exceeds the maximum number of distinct conic nodes a surface of that order can have. The existence of the surfaces is shown to depend upon that of plane curves with distinct double points.

12. Professor Nathan Altshiller-Court: On four mutually orthogonal circles.

Four mutually orthogonal circles are considered in their relations to the triangles formed by their centers and to the circumcircles of these triangles. Among others the following results are obtained. The square of the radius of any one of four given mutually orthogonal circles is equal to one half of the power of its center with respect to the circle passing through the centers of the remaining three circles. The algebraic sum of the squares of the radii of the given circles is equal to the square of the diameter of the circle passing through the centers of three of the given circles. Each of the given circles is the conjugate circle of the triangle formed by the centers of the remaining three circles, and, conversely, the conjugate circles of an orthocentric group of four triangles are mutually orthogonal. The trilinear pole of an axis of similitude of three of the given circles with respect to the triangle formed by their centers coincides with the pole of this line with respect to the fourth circle.

13. Professor J. H. Weaver: On certain points associated with a triangle.

The author has discussed the properties of four points associated with a triangle and defined as follows. Let triads of circles be drawn in such a way that the internal segments described on $B C, C A, A B$ respectively contain angles (1) $C, A, B$; (2) $B, C, A$; (3) $2 C, 2 A, 2 B$; (4) $2 B, 2 C, 2 A$. These four triads of circles determine four points, $P_{1}, P_{2}, P_{3}, P_{4}$. Among the interesting properties of these four points the following may be noted: (1) the pedal triangles of the four points are similar; (2) the pairs of lines $B P_{1}$ and $C P_{2}, C P_{1}$ and $A P_{2}, A P_{1}$ and $B P_{2}$ are parallel; (3) if $B P_{2}$ and $C P_{1}$ intersect in $A_{1}, C P_{2}$ and $A P_{1}$ intersect in $B_{1}$, and $B P_{1}$ and $A P_{2}$ intersect in $C_{1}$, then the triangles $A B C$ and $A_{1} B_{1} C_{1}$ are congruent and have the sides $A B$ and $A_{1} B_{1}, B C$ and $B_{1} C_{1}, C A$ and $C_{1} A_{1}$ respectively parallel; moreover, the points $P_{1}$ and $P_{2}$ bear the same relation to each of the triangles $A B C$ and $A_{1} B_{1} C_{1} ;(4)$ if the points $P_{3}$ and $P_{4}$ replace $P_{1}$ and $P_{2}$ in (3), then three points $A_{2}, B_{2}, C_{2}$ are determined. These three points and the points $P_{3}$ and $P_{4}$ lie on a circle.

\section{Professor Harold Hotelling: Manifolds of orthogonal}

\section{ennuples.}

Regarding an orthogonal ennuple as a set of $n$ mutually perpendicular non-sensed and indistinguishable lines through a point in $n$-space, the set of orthogonal ennuples at a point constitutes a manifold of $n(n-1) / 2$ dimensions. This paper deals with certain analysis situs properties of these manifolds. 


\section{Professor F. R. Sharpe: Superabundant nets of plane curves.}

In this paper, the author considers a general method of finding superabundant nets of plane curves which determine, by their intersections, involutions of the order of the grade. The involutions are first found on a rational surface $F_{n}$, by means of its intersections with the lines of a rational congruence $C$, having a fundamental line $l$. If $l$ is $r$-fold on $F_{n}$, the involution is of order $n-r$. A net of surfaces $N$ can be found such that any two surfaces of $N$ meet in a line of $C$. The surfaces of $N$ meet $F_{n}$ in a net of curves. When $F_{n}$ is mapped on a plane, the corresponding net of curves determines an involution of order $n-r$. By considering surfaces of orders 3 and 4, various new types of involutions of order 3 can be found, and similarly from surfaces of orders 4,5 , and 6 , involutions of order 4 .

\section{Professor V. G. Grove: Nets with equal $W$ invariants.}

Nets with equal $W$ invariants the author has called $I$ nets. A conjugate $I$ net is an isothermally conjugate net. By the introduction of the term "equal point invariants of the second kind," and by extending the idea of equal point invariants, many theorems on isothermally conjugate nets become true for $I$ nets. Conjugate nets have equal point invariants of the second kind.

17. Professor G. A. Miller: Substitutions which transform a regular group into its conjoint.

This paper appears in full in the present number of this Bulletin.

\section{Professor C. G. Latimer: A note on a theorem of Her-} mite.

If $\left|a_{i j}\right|,(i, j,=1,2,3)$, is the general symmetric third-order determinant, and $A_{i j}$ is the cofactor of $a_{i j}$, Hermite showed that the form $\phi(x)=x_{0}{ }^{2}+\sum_{i, j=1}^{3} A_{i j} x_{i} x_{j}$ repeats under multiplication. It may be shown that by a unitary transformation $T, \phi$ may be transformed into $\psi=x_{0}{ }^{2}+b c u^{2}$ $+a c v^{2}+a b w^{2}$, where $a, b, c$ are rational functions of the $a_{i j}$. Then by use of a recent result of Bell's on the product of two such forms, and by application of the transformation $T^{-1}$, we obtain Hermite's formulas for the product $\phi(x) \cdot \phi(y)$. Our proof with that of Bell's result referred to above is substantially shorter than Hermite's original proof or that of Bachmann.

19. Professor C. G. Latimer: On the representation of integers by certain indefinite quaternary forms.

In this paper it is shown that if $\alpha$ is a positive integer $\leqq 163$ in the form $4 k+3$, or the double of such an integer, and contains no square factor, then the form $f(x, y, z, w)-x^{2}+y^{2}-\alpha z^{2}-\alpha w^{2}$ represents all integers. The proof is by the method of descent, being a generalization of a method used by Dickson in obtaining similar results for the case $\alpha=-7$. It is shown 
that if $f(X, Y, Z, W)=m n \neq 0$, then $m$ is represented by $f$ if the same is true of $n$. Repeated application of the above leads to the cases $n= \pm 1$, which are easily treated. If $m$ is a prime, the existence of integers $X, Y, Z, W, n \neq 0$ such that $f(X, Y, Z, W)=m n$, is well known. Since $f$ repeats under multiplication, and since $f$ represents -1 , for each of the above values of $\alpha$, as may be readily verified, our result follows.

20. Professor L. E. Dickson: All positive integers are sums of values of a quadratic function of $x$.

This paper appears in full in the present number of this Bulletin.

21. Professor L. E. Dickson: Generalizations of the theorem of Fermat and Cauchy on polygonal numbers.

This paper will appear in full in an early number of this Bulletin.

22. Professor L. E. Dickson: Extended polygonal numbers.

This paper will appear in full in an early number of this Bulletin.

23. Professor L. E. Dickson: Generalized polygonal numbers.

These numbers include both the polygonal and extended polygonal numbers. Hence they are the values of $e(x)$ for all positive, negative, and zero integers $x$. Every integer is a sum of three generalized pentagonal numbers; also of three generalized hexagonal numbers; also of four generalized octagonal numbers; etc.

24. Professor A. J. Kempner: On the use of certain types of formulas in the theory of prime numbers.

A number of analytic expressions are known for the representation of prime numbers (by means of the gamma function, or infinite series, or definite integrals, or repeated limiting processes). None of these formulas has so far proved to be of value in the theory of prime numbers. A simple analysis of these formulas shows that they are practically all equivalent to Wilson's theorem, which is itself no deep lying theorem. It is therefore not to be expected that the mere formulation of such theorems, unless they be accompanied by a powerful analytic method of attack, will add to our knowledge in the theory of prime numbers. Such methods of attack are not known for any of the formulas.

25. Mr. W. E. Roth: A solution of the matric equation $P(X)=A$.

This paper is concerned with finding, by elementary methods, such solutions of the equation $P(X)=A$ as are expressible as polynomials in $A$. Here $P(X)$ is a polynomial in $X$ with scalar coefficients, and $A$ is a square matrix of order $n$, not necessarily non-singular. Such solutions were found by Frobenius (Berliner Sitzungsberichte, 1896) for the case 
$X^{2}=A$; they are given by Dickson in his Modern Algebraic Theories, for the case $X^{m}=A$. In both cases, $A$ is supposed to be non-singular. The equation $X^{m}=A$ has also been treated by Cecioni (Annali della Reale Scuola Normale, Pisa, 1909), and by Kreis (Vierteljahrschrift, Zurich, 1908); in his Zurich dissertation, the latter author had also studied the general equation $P(X)=A$. In all of the papers cited, use is made of the Weierstrass elementary divisors and associated normal forms; the method is not easy to follow and is complicated in its application.

\section{Mr. E. R. Heineman: Generalized Vandermonde} determinants.

A generalized Vandermonde determinant is obtained from the ordinary Vandermonde determinant by permitting the indices to take any set of values. The Vandermonde matrix is defined to be the Vandermonde determinant with the $n$th powers of its variables added as an extra row. By successively blocking out each of the first $n$ rows of this matrix, we obtain $n$ determinants which can be called secondary Vandermonde determinants. The ordinary Vandermonde determinant, which we get by omitting the last row of this matrix, will be called the principal Vandermonde determinant. It can be shown that every generalized Vandermonde determinant is expressible as a determinant-function of the principal and secondary Vandermonde determinants.

27. Professor H. S. Vandiver: On the theorem of Kummer concerning power characters of units in a cyclotomic field.

This paper presents, in a modern form, the argument used by Kummer in Crelle's Journal (vol. 44 (1852), pp. 121-130) for obtaining expressions giving the $p$ th power characters of units in the field defined by a primitive $p$ th root of unity, $p$ being an odd prime. Extensive use of these results will be made in other papers by the author.

28. Professor H. S. Vandiver: Summary of results and proofs concerning Fermat's last theorem. (Third note.)

In this note, among other results a proof is indicated for the following theorem: Under the assumptions (1) none of the Bernoulli numbers $B_{\nu p}(\nu=1,2,3, \cdots,(p-3) / 2)$ is divisible by $p^{3}$, and (2) the second factor of the class number of the field $k(\alpha), \alpha=e^{2 i \pi / p}$, is prime to $p$, it follows that $x^{p}+y^{p}+z^{p}=0$ is not satisfied in rational integers $x, y$, and $z$, prime to each other, none zero, if $x y z \equiv 0(\bmod p)$, and $p$ is an odd prime.

\section{Professor H. S. Vandiver: On an extension of the Ber- noulli summation formula.}

In this note is considered a theorem of Glaisher's which expresses in terms of Bernoulli numbers the sum of $n$th powers of integers in an arithmetical progression. 
30. Professor H. S. Vandiver: On the norm-residue symbol in the theory of cyclotomic fields.

This symbol (Hilbert, Bericht, p. 413) has always, apparently, been used to represent a summation. In the present paper it is shown how the summation may be carried out.

31. Professor C. C. Camp: A multiple-parameter differential system which leads to a development of a function in $p$ variables.

The system $u_{i}^{\prime}+\left(\sum_{j=1}^{p} \lambda_{j} a_{i j}\right) u_{i}=0, i=1,2, \cdots, p$, with the boundary conditions $u_{i}(\pi)=u_{i}(-\pi), i=1,2, \cdots, p$, where $a_{i j}$ is an integrable function of $x_{i}$ either identically zero or of constant sign and such that the determinant $\left|A_{i j}\right| \neq 0,2 \pi A_{i j} \equiv \int_{-\pi}^{\pi} a_{i j} d x_{i}$, leads to a multiple expansion in which the coefficients involve the $p$-fold integral of $f\left|a_{i j}\right| \prod_{i=1}^{p} v_{i}^{*}$; $v_{i}{ }^{*}, i=1,2, \cdots, p$, being a principal solution of the adjoint system. We make the change of parameters $\nu_{i}=\sum_{j=1}^{p} \lambda_{j} A_{i j}$ for the convergence proof, and in the first integrations by parts in the contour integrals we replace the elements of the first column of $\left|a_{i j}\left(s_{i}\right)\right|$ by $\sum_{j=1}^{p} \lambda_{j} a_{i j}\left(s_{i}\right)$. In certain subsequent integrations we have the cofactors of these elements as multipliers in the integrands. By replacing $\lambda_{k}$ by its appropriate values $\left(\nu_{i}-\sum_{j=1, j \neq k}^{p} \lambda_{j} A_{i j}\right) / A_{i k},(i=1,2, \cdots, p ; k=2,3, \cdots, p)$, in the several integrands of the exponential indices, we may evaluate the limits of the parts which do not contribute zero as the so-called mean value of $f$.

32. Professor A. S. Besicovitch: Fundamental geometric properties of linearly measurable plane sets of points.

The notion of a linearly measurable plane set of points was introduced in 1914 by Carathéodory. These sets $(A)$ include as a special case rectifiable plane curves. The present paper develops a general theory of such sets. The notion of the density and of the tangent are introduced and discussed. The sets $(A)$ are classified as regular and irregular. The regular sets $(A)$ in many respects are analogous to the rectifiable curves. For instance, the tangent exists at almost all points of a regular set $(A)$; to any regular set $(A)$ of measure $L$ and any positive number $\epsilon$, there corresponds a set (finite or denumerable) of rectifiable curves, of total measure $<L+\epsilon$, which contains almost all the points of $(A)$. On the other hand, no such properties exist for the irregular sets $(A)$. They are entirely dissimilar to the rectifiable curves, which is shown by many examples. In one of these, an irregular set $(A)$ is constructed, of measure $>0$, whose projection on any straight line has measure 0 .

33. Professor Harold Hotelling: Correlations with incomplete data.

The data for a correlation problem consist essentially of a matrix of observations. One row represents each variate, and one column each instance. Ordinary, partial, and multiple correlation coefficients are all 
invariant under certain operations on the matrix. Frequently incompleteness of data leaves certain elements of the matrix unknown. In such cases statisticians have faced the dilemma of discarding every column containing an unknown element, which may so far reduce the number of instances as to rob the results of significance, or of using all possible instances for calculating each ordinary correlation, which may give impossible values for the multiple and partial correlations. But regarding the data as obtained by a random sampling of a normal distribution, it is possible to escape both horns of the dilemma in determining the most likely values, which are necessarily consistent, in the aggregate sampled. The equations for doing this are presented. By similar means an explicit solution is obtained for the following problem. Two variables are known a priori to be uncorrelated, but in a sample there is usually some correlation. What corrections should on this account be applied to the observed correlations of these variables with others, and to partial and multiple correlations involving them?

\section{Professor Harold Hotelling: Spaces of statistics and} their metrization.

An ordinary statistical graph is a geometric figure whose essential properties are invariant only under a group of transformations even more limited than that of euclidean geometry. However, certain statistical problems admit of geometric representation in a manner for which the concepts of differential geometry have significance. One way of metrizing the space of statistical variables is by means of the quadratic form appearing in the normal law of error. This becomes a differential form in various problems in evolution and in economics. Examples of the latter are given in a paper by the author in the September, 1927, number of the Journal of the American Statistical Association.

35. Professor A. J. Kempner: On the shape of polynomial curves. (Second paper.)

This is a continuation of a paper presented to the Society in 1919 under the same title. In the earlier paper, the problem treated was to prove the existence of polynomial curves $y=a_{0} x^{n}+\cdots+a_{n}$ (all quantities involved real) with a prescribed arrangement (arbitrary except for obvious restrictions) of the order of magnitude in which the successive maxima and minima are arranged. In that paper, points of inflection were not assumed on the curves. In the present paper, by considering them as being generated by letting successive maxima and minima coincide, with subsequent further continuous variation of the coefficients, points of inflection are taken into account.

36. Professor H. E. Buchanan: On the oscillations of three finite masses near the equilateral triangle solutions.

In this paper the author discusses the equations of variation near the equilateral triangle positions. The characteristic exponents are found, and 
their nature depends on a certain relation between the masses. In case one of the masses is infinitesimal, these exponents reduce to those given by Moulton in his celestial mechanics. The instability of the equilateral triangle positions is proved, and two types of periodic orbits are shown to exist.

37. Professor H. E. Buchanan: Note on a certain memoir of Liouville's.

In a very interesting paper published in Connaissance des Temps, 1845, Liouville discusses the stability of the straight-line solutions of the threebody problem. His result depends on the solution of the equation

$$
\alpha^{4}+\left(2 n^{\prime 2}-n^{2}\right) \alpha^{2}+\left(n^{\prime 2}-n^{2}\right)\left(2 n^{2}+n^{\prime 2}\right)=0 .
$$

He shows that $n^{\prime 2}-n^{2}$ is negative for all values of the masses, and consequently the straight line solutions are unstable because of the presence of a term of the type $e^{\alpha t}, \alpha$ real and positive. In the present note it is shown that Liouville's equation is exactly the same as an equation of Buchanan's in a paper in the American Journal of Mathematics (vol. 45 (1923)), p. 98, equation 12), where the periodic orbits near the straight-line solution are discussed, except that Liouville always keeps his three bodies in a straight line. It follows that Liouville's equation is the same as Moulton's Periodic Orbits, Carnegie Institution Publication No. 161, and Plummer's Monthly Notices, Royal Astronomical Society, vol. 62 (1901), if one of the masses is infinitesimal.

38. Professor L. M. Graves: Discontinuous solutions in space problems of the calculus of variations.

The extension of the Jacobi condition to the case of minimizing curves with corners, the so-called discontinuous solutions, was given by Caratheodory for problems in the plane. His condition is apparently not extensible to space problems, and the details of the proof are very complicated for the plane case. The new form of the condition of Caratheodory obtained by the present author states that the functional determinant of the family of "extremaloids" or broken extremals, whose zeros correspond to points of contact with the envelope of the family, shall not change sign at a corner of a minimizing curve. The statement and proof are practically as simple for $n$-dimensional problems as for the plane problem. When modified in the usual way, the new condition fits into the familiar set of sufficient conditions for a minimum.

\section{Professor D. C. Gillespie: On infinite determinants and their associated systems of linear equations.}

If in a normal determinant each element of the principal diagonal be diminished by unity, the resulting double array of elements are the terms of an absolutely convergent double series. The normal determinant converges; if its value is different from zero and the constant terms in the 
associated system of equations form a bounded set, the equations have one and only one bounded solution. The striking fact that the solution obtained is the only bounded solution is, of course, due to the assumption made concerning the terms of the principal diagonal. The determinants considered in this paper have in common with normal determinants the property that the elements of the principal diagonal approach unity, but differ from them in that the absolute value of the elements in diagonals immediately below the principal diagonal are allowed to approach unity. The determinants are shown to converge, and a solution is obtained for the system of equations associated with each non-vanishing determinant, the solution being the only bounded one if a bounded solution exists. If no bounded solution exists, the solution found is the only solution in which the $n$th unknown is less in absolute value than the $n$th term of a certain sequence.

\section{Professor G. Y. Rainich: Deviation from normality of congruences of curves.}

For a congruence of "equidistant" curves in curved $n$-dimensional space certain expressions which vanish in the case of a normal congruence are introduced as giving in the general case a measure of deviation from normality in the neighborhood of a curve. Integrals of these expressions (which represent an antisymmetric tensor) over a surface cutting the curves of the congruence are introduced, as giving the total deviation for the corresponding portion of the congruence. Conditions are considered (for $n>3$ ) under which this total deviation vanishes over a closed surface. In the case for which $n=5$, the Kaluza-Klein interpretation of electromagnetism is obtained. The centrosymmetric case is worked out as an example of the general theory.

\section{Professor D. V. Widder: On the expansion of analytic} functions of the complex variable in generalized Taylor's series.

In a previous paper the representation of a real function in generalized Taylor's series was discussed. The methods there employed are not applicable to the complex case. In the present paper new methods are developed and the representation of an arbitrary analytic function is obtained. The result is comparable with a result of Birkhoff's given in the Comptes Rendus, 1917.

42. Mr. J. J. Gergen and Professor D. V. Widder: On Taylor's series admitting the circle of convergence as a singular curve.

This paper obtains a new criterion that the circle of convergence of a power series be a singular curve. The criterion is a generalization of one recently given by $\mathrm{S}$. Mandelbrojt. By an application of the result, an elementary proof of a familiar theorem on lacunary series is given. 


\section{Professor R. L. Moore: A separation theorem.}

In this paper the following theorems are proved. (I) If, in a plane $S$, $M$ is a bounded continuous curve, $K$ is a closed subset of $M$, and $H$ is a subset of $K$ and of some connected subset of $K+(S-M)$ but no point set of which $H$ is a proper subset satisfies these conditions, then if the continuum $E$ is a subset of $K-H$, there exists a simple closed curve lying wholly in $M-K$ and separating $H$ from $E$. (II) If, in a plane $S, K$ is a closed subset of a continuous curve $M$, and $A$ and $B$ are two points of $M$, then if $A$ and $B$ lie in a connected subset of $K+(S-M)$ they lie in a closed and connected subset of $K+(S-M)$. (III) In order that a bounded continuum $M$ in a plane $S$ should be a continuous curve, it is necessary and sufficient that if $t$ is an arc and $H$ is an open subset of $M+t$, and $A$ and $B$ are points of $S-H$ which are weakly separated by $H$, then $H$ contains a simple closed curve that separates $A$ from $B$. The first of these theorems may be regarded as an extension of a theorem of Zoretti's.

44. Mr. J. L. Dorroh: Concerning a set of metrical hypotheses for geometry.

In his paper Sets of metrical hypotheses for geometry (Transactions of this Society, vol. 9 (1908), pp. 487-512) R. L. Moore raises the question whether it follows from the set $O$ of order axioms and the set $C$ of congruence axioms employed therein that every segment has a mid-point. In the present paper this question is answered in the affirmative.

\section{Dr. G. T. Whyburn: On a problem of W. L. Ayres.}

The author shows that if a plane continuous curve $M$ does not contain more than a finite number of simple closed curves of diameter greater than any preassigned positive number, then every connected subset of $M$ is arcwise connected. This answers a question raised by W. L. Ayres in his paper Concerning continuous curves of certain types. (See abstract in this Bulletin, vol. 32 (1926), p. 307; the paper will appear in Fundamenta Mathematicae.) In proving this proposition it was shown that every continuous curve satisfying the hypothesis of this theorem has the property that each of its maximal cyclic curves contains only a finite number of simple closed curves. Then with the aid of the author's theorem that every connected subset of a continuous curve is arcwise connected provided that every connected subset of each of its maximal cyclic curves is arcwise connected (see my paper Concerning the structure of a continuous curve, presented to the Society Dec. 31, 1926; offered to the American Journal), the proof is easily completed.

\footnotetext{
46. Dr. G. T. Whyburn: Concerning the cut points of continua.

If $X$ is any point set, let $X^{*}$ denote a subset of $X$ obtained by omitting from $X$ some countable set of points. Let $M$ be any plane continuum, and $G$ the set of all the cut points of $M$. Then (1) if $H$ is any uncountable subset
} 
of $G$, there exist two points of $H$ which are separated in $M$ by uncountably many points of $H$; (2) there exists a set $G^{*}$ (possibly null) such that for each point $X$ of $G^{*}, M-X$ is the sum of two connected point sets; (3) every collection of mutually exclusive subcontinua of $M$ each of which contains at least one point of $G$ is countable; (4) for every subset $E$ of $G$ there exists an $E^{*}$ no point of which belongs to any subcontinuum of $M$ containing no other point of $E$; (5) there exists a $G^{*}$ every point $X$ of which is an end point of every continuum obtained by adding $X$ to some maximal connected subset of $M-X ;(6)$ there exists a $G^{*}$ such that $M$ is connected im kleinen at every point of $G^{*}$; (7) there exists a $G^{*}$ such that every subcontinuum $N$ of $M$ is connected im kleinen at every point of $G^{*} \cdot N$; (8) there exists a $G^{*}$ every point of which is a point of Menger order two of $M$.

47. Dr. G. T. Whyburn: Concerning certain kinds of continuous curves and other continua.

The following theorems are proved. (1) Every connected and relatively open subset of an arcwise connected im kleinen point set is arcwise connected. (2) If $H$ denotes the sum of the boundaries of all the complementary domains of a continuous curve $M$ every subcontinuum of which is a continuous curve, then (a) $H$ is arcwise connected, and (b) $M-H$ is totally disconnected. (3) If every maximal cyclic curve of a continuous curve $M$ is a simple closed curve, then $M$ is a Menger "regular curve" and, indeed, for any $\epsilon>0, M$ is the sum of a finite number of continua each of diameter $<\epsilon$ and no two of which have more than one point in common. The case where $M$ is the boundary of a domain is a special case of (3). (4) Every "regular point" (Menger) of a continuum $M$ is regularly accessible from every complementary domain of $M$ to the boundary of which itbelongs. (5) Every cut point of a continuum $M$ which is of Menger order two belongs to the boundary of only one complementary domain of $M$. (6) If every point of a continuum $M$ which contains no domain is regularly accessible from $S-M$, then $M$ is a Menger regular curve.

48. Dr. W. L. Ayres: Concerning subsets of a continuous curve which can be connected through the complement of the continuous curve.

A subset $N$ of a plane continuous curve $M$ is said to be connected through $N$ and $S-M$ if there exists a subset $H$ of $S-M$ such that $N+H$ is connected. (1) If $K$ is a bounded subcontinuum of $M, T$ is a totally disconnected cutting of $K$, and $x$ and $y$ are points of $K-T$ which cannot be connected through $K-T$ and $S-M$, then there exists a simple closed curve $J$ which is a subset of $M$, separates $x$ and $y$, and is such that $J \cdot K$ is a subset of $T$. (2) A bounded $M$-domain is simply connected with respect to $M$ if and only if its $M$-boundary is connected through $B$ and $S-M$. (3) If $K$ is a closed cutting of $M$ such that if $H$ is any component of $K$ then $K-H$ is not a cutting of $M$, then $K$ is connected through $K$ and $S-M$. (4) In order that a plane continuum $K$ be a continuous curve it is sufficient 
that if $A$ is any arc, $H$ is a closed subset of $K+A$, and $x$ and $y$ are two points of $H$ which can be connected through $H$ and $S-K$, then $x$ and $y$ can be connected through $H$ and $S-K-A$ by a continuum. (R. L. Moore has shown the necessity of (4).)

49. Professor W. A. Wilson: On irreducible cuts of the plane between two points.

If $F$ is an irreducible cut of the plane between two points, any component of the complement of $F$ which has $F$ as its frontier is called a principal component. A decomposition of a continuum $C$ into two subcontinua is called irreducible if $C$ is not the union of one of the subcontinua and a proper subcontinuum of the other. The following theorem is obtained. Let $F$ be a bounded irreducible cut between two points which is decomposable and let $F=H+K$ be an irreducible decomposition of $F$ into proper subcontinua. According as the number of principal components of the complement of $F$ is finite or infinite, $H \cdot K$ is the sum of the same number or an infinite number of closed subsets, no pair of which have common points, and $H$ and $K$ are both irreducible between each pair of these sets. This is an analogue of C. Kuratowski's theorem regarding a completely irreducible cut of the plane, i.e., a cut of the plane which is the frontier of every component of its complement.

50. Dr. C. H. Langford: An analysis of some general propositions.

This paper appears in full in the present number of this Bulletin.

51. Professor J. L. Walsh: On the approximation to harmonic functions by harmonic polynomials.

This paper has appeared in the September-October number of this Bulletin.

52. Professor J. L. Walsh: On the expansion of analytic functions in series of polynomials and of other analytic functions.

This paper presents some extensions of the author's previous work on expansions of arbitrary functions in terms of polynomials (Transactions of this Society, vol. 26 (1924), pp. 155-170). In particular, there are considered the expansion of a discontinuous function, the analog of Gibbs's phenomenon, the analog of Abel's theorem and its converse, and equivalence of expansions not merely on a single curve but on a oneparameter family of curves.

53. Dr. G. M. Merriman: On sufficient conditions for the convergence and Cesàro summability of the allied series of a double Fourier series.

This paper has appeared in the September-October number of this Bulletin. 


\section{Miss Florence M. Mears: Riesz summability for double} series.

This paper defines for double series a mean, $(R: \lambda, p ; \mu, r)$, corresponding to the Riesz definition, $(R, \lambda, p)$, for summing simple series. It includes theorems relative to the regularity and total regularity of the extended definition; it establishes a relation between methods of summation of the same type, $(\lambda, \mu)$, when either $p$ or $r$, or both $p$ and $r$, are changed; a relation between methods of summation of the same order, $(p, r)$, when either $\lambda$ or $\mu$, or both $\lambda$ and $\mu$, are changed; certain necessary conditions for the Riesz summability of double series; theorems for the Dirichlet and Cauchy products of double series corresponding to those of Mertens, Cauchy and Abel for the Cauchy product of simple series; a sufficient condition for the summability of the product of two double series to the correct sum.

55. Dr. W. M. Whyburn: Existence and oscillation theorems for non-linear differential systems of the second order.

The method of successive approximations is used to establish a general existence theorem for the system of first-order differential equations $d y_{i} / d x=\sum_{i=1}^{n} A_{i j}\left(x, y_{1}, \cdots, y_{n}\right) y_{i},(i=1,2, \cdots, n)$. The second-order system $d y / d x=K(x, y, z ; \lambda) z, d z / d x=G(x, y, z ; \lambda) y$, is considered along with boundary conditions of the type $y(a, \lambda)=y(b, \lambda)=0$. Existence and oscillation theorems are established for cases where $K$ and $G$ satisfy conditions similar to those imposed in treating the ordinary linear systems of the Sturmian type. A treatment of linear Sturmian systems is given in which several of the usual conditions on the coefficients are replaced by symmetric conditions on $K$ and $G$. The methods of this paper are based on transformations of the type $y=u \cos v, z=u \sin v$.

56. Professor W. P. Webber: On a generalization of the Weierstrassian theory of elliptic functions.

Defining the variable $u$ by the vector equation $u=i_{1} x_{1}+i_{2} x_{2}+\cdots+i_{p} x_{p}$, where $i_{1}, i_{2}, \cdots, i_{p}$ are unit vectors in the directions of a system of mutually perpendicular axes $O X_{1}, O X_{2}, \cdots, O X_{p}$, and $x_{1}, x_{2}, \cdots, x_{p}$ are real coordinates of a point referred to these axes; defining $w$ by the vector equation $w=2 i_{1} m_{1} \omega_{1}+2 i_{2} m_{2} \omega_{2}+\cdots+2 i_{p} m_{p} \omega_{p}$, where $m_{1}, m_{2}, \cdots, m_{p}$ take all integer values independently and where $\omega_{1}, \omega_{2}, \cdots, \omega_{p}$ are arbitrary real constants; and employing a generalization of ordinary vector algebra, the author constructs the function

$$
F_{p}(u)=\left(1 / u_{p}\right)+\sum^{\prime}\left[1 /(u-w)^{p}-1 / w^{p}\right],
$$

which is valid and possesses $p$ periods $2 \omega_{1}, 2 \omega_{2}, \cdots, 2 \omega_{p}$; the accent on $\sum$ indicates that $w=0$ is omitted. Convergent series valid in the fundamental cell are obtained for the cases $p=3$ and $p=4$. Functions analogous to the classical functions $\sigma, \zeta, Z$ are given.

57. Professor G. D. Birkhoff: Reversibility in dynamics.

In this paper it is proved that a necessary and sufficient condition for complete stability of a periodic motion is the reversibility of the time $t$ 
in the differential equations, provided that the dependent variables are also subject to transformation.

58. Professor G. D. Birkhoff: The periodic motions near a stable periodic motion of a dynamical system.

On the basis of a very simple lemma concerning the invariant points of a transformation of the plane in the neighborhood of a given invariant point, the author shows that there exist infinitely many periodic motions in an arbitrarily small neighborhood of a stable periodic motion of a dynamical system with two degrees of freedom. This lemma is closely related to the extended form of Poincaré's last geometric theorem.

59. Professor G. C. Evans: Note on a generalization of a theorem of Bôcher.

Let $u(M)$ be a "potential function of its vector derivative or gradient" and satisfy Bôcher's equation $\int_{s} D_{n} u d s=0$ "almost everywhere" on rectangles in the plane open region $\Sigma$. Then $u(M)$ has at most removable discontinuities in $\Sigma$, and when these are removed by changing the values of $u(M)$ at most in a set of measure zero, it becomes continuous with all its derivatives and satisfies Laplace's equation at every point of $\Sigma$. The theorem remains valid if the set of rectangles is replaced by any "translatable regulat net"; and a corresponding theorem holds in any number of dimensions. Since it is not assumed that the curves on which Bôcher's equation holds are level curves of a harmonic function, an indirect proof is necessary.

\section{Professor G. C. Evans: Poisson's equation in the plane and other equations of elliptic type.}

The equation considered is $\int_{s} D_{n} u d s=\Phi(s)$, where $\Phi(s)$ is the function of curves with regular discontinuities which corresponds to a given arbitrary completely additive function of point sets $\Phi(e)$, in an open finitely connected region $\Sigma$. The principal solution is a "potential function of its vector derivative or gradient," belongs to the class (ii) of the author's book on Logarithmic Potential, and takes on zero boundary values almost everywhere "in the narrow sense." Both types of the first boundary value problem are uniquely solvable in their respective classes of functions. The corresponding problems for the sphere may also be solved. By means of integral equations, other equations of elliptic type may be treated, as in the Cambridge Colloquium. The given equation more or less reduces to Poisson's equation if $\Phi(s)$ or $\Phi(e)$ is assumed to be absolutely continuous.

\section{Dr. C. F. Roos: A mathematical theory of depreciation and replacement.}

In as much as a machine is usually replaced by another machine before its useful life has ended, it is quite important to consider the problem of determining the time at which a machine in operation should be replaced by another machine whose operating expense is different so that a maximum profit is obtained for some period of time extending from an initial time $t_{1}$ 
through a replacement time $\omega$ to some final time $t_{2}$ greater than $\omega$. When formulated as a problem in the calculus of variations, this replacement problem is a type of Lagrange problem with variable end points and discontinuous integrand. In this paper a general formulation is made of a replacement problem and a special example of an actual economic situation is solved in some detail.

62. Dr. C. F. Roos: The problem of depreciation in the calculus of variations.

This paper will appear in full in an early number of this Bulletin.

63. Professor W. J. Trjitzinsky: Expansion in series of non-inverted factorials.

Using Cauchy's result $f(z)=[1 /(2 \pi i)] \int_{C}[f(t) /(z-t)] d t$ and $\int_{0}^{1} u^{n}(1-u)^{v} d u=(n !) /[(v+1) \cdots(v+n+1)]$, we expand $1 /(z-t)$ in a series of direct factorials in $z$ uniformly convergent when $R(t)<-1$. It follows that if $f(z)$ is analytic on and outside a closed contour $C$ situated to the left of $R(t)=-1$, then for $R(z)>0$,

$$
f(z)=a_{0}+a_{1} z+a_{2} z(z-1)+\cdots+a_{n} z(z-1) \cdots(z-n+1)+\cdots,
$$

where $a_{n}=-[1 /(2 \pi i)] \int_{C}[f(t) /(t(t-1) \cdots(t-n))] d t$, and $\left|a_{n}\right|<h /(n !)$. This can be generalized for any position of $C$.

64. Professor W. J. Trjitzinsky: A class of infinite products and an application to the theory of the gamma function.

We consider functions

$$
L a_{i}(z)=\frac{e^{-\alpha z}}{z} \prod_{n=1}^{\infty} e^{z l a_{n}}\left(1+\frac{z}{a_{n}}\right)^{-1},
$$

where $\alpha$ is analogous to the Eulerian constant, and the $a_{n}$ are under certain restrictions; these functions reduce to the gamma function when $a_{n}=n$. A multiplication theorem for products $L_{a_{i}}(z) L_{a i}\left(z+p_{1}\right) \cdots L_{a i}\left(z+p_{n}\right)$ is obtained, where the $p_{i}$ satisfy certain inequalities. When $a_{i}=i$, this theorem yields a multiplication theorem for the gamma function that is disctinct from the classical theorem.

65. Dr. W. L. Ayres: Concerning continuous curves in $n$ dimensions.

In this paper the following theorems which have been proved previously for two dimensions are proved for $n$ dimensions: (1) in order that a point $P$ of a continuous curve $M$ be an end point of $M$ it is necessary and sufficient that $P$ be a non-cut point which lies on no simple closed curve of $M$; (2) in order that a continuous curve be cyclically connected, it is necessary and sufficient that it contain no cut point; (3) in order that there exist a point which separates the points $A$ and $B$ in the continuous curve $M$ it is necessary and sufficient that every two arcs of $M$ whose end points are $A$ and $B$ have an interior point of both in common. 
66. Professor L. E. Dickson: Complete solution of the Waring-Kamke problem on sums of integral values of quadratic functions.

Let $q(x)$ be an integer $\geqq 0$ for every integer $x \geqq 0$. For every $s \geqq 5$, there is found the least integer $e_{s}$ such that every positive integer is a sum of $s$ values of $q(x)$ and $e_{s}$ numbers 0 or 1 . There is deduced the least value $L$ of $s+e_{8}$ for $s \geqq 5$. Next, it is shown that $4+e_{4} \geqq L$ except for specified special cases, and then $4+e_{4}=L-1$. Except for these special cases, it is not necessary to carry out the very difficult work of finding the exact value of $e_{4}$. By the new method developed, it is relatively simple to find each $e_{s}$ for $s \geqq 5$. The memoir giving the complete solution of this problem will appear in the American Journal.

67. Professor J. V. Uspensky: On the number of representations of integers by certain ternary quadratic forms.

Denoting by $(a, b, c)$ the form $a x^{2}+b y^{2}+c z^{2}$, the author gives the expression for the number of representations of integers by the following forms: $(a, b, c)=(1,2,3),(1,2,6),(2,3,6),(1,3,6),(1,1,3),(1,3,3)$, $(1,1,6),(1,6,6),(1,1,5),(1,5,5),(1,2,5),(1,5,10),(1,2,10)$, $(2,5,10)$.

68. Professor J. V. Uspensky: On the connections between numbers of representations of integers by two totally different quadratic forms.

For certain couples of quadratic forms involving three and four variables without any apparent relationship to each other, there exist unexpected relations between the numbers of representations of integers by these forms. The author gives several instances of this rather remarkable phenomenon.

69. Professor Arnold Emch: On the mapping of the sextuples of the symmetric substitution group $G_{6}$ in a plane upon a quadric.

This paper appears in full in the present number of this Bulletin.

R. G. D. Richardson, Secretary. 